Pursuing a course, in dealing with the effect of distance on the left hand: similar to that which gave us Fig. 3 for the effect of colors, we get Fig. 4. It will be seen from Fig. 4 that the proportion of responses with the left hand increases up to the 13 -inch distance, which is represented by 100 , and then declines rapidly. From this it is seen that the effect of distance is not exactly what we should expect, but the fact that various colors were the stimuli probably renders this last question too complex to be answered by the experiments we have been dealing with. To answer this question properly it appears best to take a large number of experiments with a single object.

Rufus E. Marsden.

\title{
EXPERIMENTS IN THE PERCEPTION OF THE THIRD DIMENSION.
}

I have frequently had opportunity to experiment at random on the perception of solidity, and as the results exhibit some bearing upon the study of the so-called motor factors in perception these results may be described.

The experiment which I have tried many times, and especially when travelling, which affords good opportunities for the experiments, consists in testing both binocular and monocular vision in the way described below.

It is perhaps often remarked that favorable conditions will enable the observer to see in the opposite direction from which the objects are situated the images of any given thing, as if seen through the solid matter toward which the observer is actually looking. Changes of adjustment will affect the results somewhat. That is, adjustment to the distance necessary to fuse images from the real objects in the actual field of vision will prevent the perception of objects with reflected images from the opposite direction. But adjustment to the apparent objects will prevent the perception of the real objects in front of the eyes, so that the effect is actually to destroy for purposes of actual perception the real world at which we look, and we see the imaginary world through it, as if vision could perform the miracle of the Röntgen rays. In this condition a very curious phenomenon occurs. Let me describe a concrete case.

Suppose yourself facing the direction in which the cars are moving, and the light effects are favorable to your perceiving objects reflecting their images from the left to the window panes to your right, so that you apparently see the objects, say houses, to the right on the 
landscape or other houses at which you are actually looking. These houses may appear to be variously located to other observers. But for me there is a perfectly uniform action in this respect. If the eyes are adjusted to fuse the images of the houses on my left I see them through the landscape or houses toward which I am looking. I may even so concentrate attention as to become absolutely oblivious of the real houses, and the only world that appears to have any reality at all is the imaginary houses or object at which I am apparently looking. Only when I change the adjustment to the images of the real houses or adapt attention to them do I become conscious of them at all, except as vague images in the field of vision. Now what $I$ always notice in the experiment is that the localization of both the real and imaginary world, is definitely related to the different degrees of convergence necessary to determine fusion. The real world, if situated so that this fusion occurs with a greater degree of convergence than the imaginary requires, appears nearer. If not nearer in fact the imaginary world appears nearer. But take the former condition. We have localization proportioned in some way to the degree of convergence, not at which it is at the time, but at which it must be for fusion of images outside the given degree for actual fusion. Thus, although the motor conditions are the same for both perceptions the localization is different. I have called attention in experiments made years ago to this phenomenon. What I wish to note now is a different fact, though it has the same meaning. I have described the binocular effect, and this indicates a difference of perspective for the real and imaginary worlds. But if, while the eyes are adjusted binocularly for the fusion of the images in the imaginary world, I close one eye, the localization, while I remain perfectly still, is the same for both the real and the imaginary worlds. That is, they are seen in the same plane, when monocularly seen. The perspective which is so clearly seen in binocular vision to be different for the different sets of images is now seen to be the same for both. Whether the motor conditions have been changed or not makes no difference in the explanation of the phenomena. If those conditions are altered by closing one eye, and the convergence is probably altered somewhat, the result remains the same. If they are not altered the result also remains the same.

But now, if we move the head from side to side, while keeping one eye closed and using only monocular functions, thus imitating the effects of motion in the cars, we shall notice that the perspective of binocular vision springs at once into effect again. That is, the real and the imaginary worlds seem differently localized and no longer to 
lie in the same planes. It is to be noticed, again, that there is no difference of motor conditions for the perception in this case. The motion of the head can hardly be appealed to, as this does not ordinarily affect the results of vision. The adjustment of the monocular system remains the same for both the real and imaginary worlds, and yet the difference of localization is as distinct as in binocular perception. We have a parallax of motion, as we may call it, but this represents only the difference in velocity of the images on the field of vision, caused by the movement of the head, while the muscular adjustment for the focusing of the eyes on the objects is not of the kind to account for the difference of perspective, as that muscular condition remains common to the perception of both sets of images. The deciding characteristic is the parallax of motion, and the effect carries no appearances of being a mere inference. It is as distinct and fixed as any form of localization is, where inference is not suspected or believed. The effect thus has every credential of an organic function of sone sort whether we choose to regard it as sensory or motor. It represents in all its characteristics a constitutional function. At the same time, as described and as it always occurs with me, I find no such coördination with what I have understood as 'motor' influences that I could treat it as so caused or as so represented in nature.

In the study of the bearing of such phenomena as I have described on the motor theory of such perceptions I wish to call attention to what seems an inherent difficulty in modern theories couched in the term 'motor.' To call space perception or any other product of similar action 'motor' may be clear or obscure according to the understanding of the term 'motor.' In contrast with sensory functions the term originally represented a wholly unconscious process, and so indicated all the difference from the sensory that the unconscious stands for in comparison with the conscious. 'Motor' thus stood for the initiation of muscular action, a function that never is conscious except in deliberate volition and even then only to the extent of the fiat which finally realizes itself in motion. In the application of the term 'sensory' thus contrasted we were to understand the actual facts of consciousness connected with our primary experiences. Thus the 'sensory' was definitely what we knew and experienced in sense perception. The 'motor' was what we did not know, but what we inferred took place in the central system somewhere. It was of an impulsive character and accounted for the various movements of the organism whether consciously or unconsciously initiated. Now in our modern enthusiasm about ' motor' explanations for various phenomena 
we constantly forget two things. First, the fact that 'motor' ana 'sensory' come to us with an implied contrast in their relation to consciousness, so that an appeal to the 'motor' invariably suggests an unconscious fact in the result which we are explaining. Secondly, the fact that we do not adequately distinguish between this traditional use of the term 'motor' to denote the unconscious agencies connected with the muscular system, and the application of the term to denote the 'consciousness of motion,' which is a sensory fact and not ' motor' in the old sense of the term. This modern use becomes identical with the 'sensory' and not contrasted with it. Consequently there is little excuse for the controversies which have centered about the various problems of space perception or any other question associated with the so-called 'motor' activities. 'Motor' to describe an activity or function wholly below consciousness is one thing, to describe a fact of which we are conscious is another and very different thing separated from the former by all the diameter of a contradiction.

To make our problem concrete. In the traditional conception of the term, to call the perception of space, or the third dimension, a ' motor' phenomenon would be to deny its sensory character and to take it out of the field of consciousness altogether. Here was where Berkeley created difficulties in his theory. The observer never detected any muscular sensations in his visual act except when straining the eyes and this indicated no apparent effect on space perception. All that he could do was to say or imply that the ' sensations' were unconscious, which was to take the phenomena outside the field of legitimate study. But for us to say that such perception is a 'motor' phenomenon and mean by it that it is either constituted by or caused in connection with the sensations of motion, is to conceive the phenomenon as a sensory one and not to contrast it with the sensory at all. The controversies which have centered about the problem have been caused by the abandonment of the original meaning of the term ' motor' while retaining its opposition to the sensory after that change This is a frequent vice of our psychologists and it leads to both their misunderstanding of the problem as conceived in relation to historical questions and their being misunderstood by others. What we want is a clearer definition of what we are talking about. I have not yet seen any such careful account of terms with a view to avoiding misconception and controversy, and I know no term more in need of careful explication than the term 'motor.'

With the differences of meaning attached to it, I think the reader can anticipate the solution to the issue about the 'motor' perception 
of space. If we mean by the 'motor' theory that the function determining space perception is of the order which initiates muscular action, we have an impossibility in the simultaneous localization of different objects in different planes. The 'motor' or muscular functions are seen, in such phenomena as I have described, to be the same or common, but the localization different. The localization varies when the 'motor' action does not, so far as that is conceived in the mechanical terms for which muscular action stands. The 'motor' theory thus seems to have an irrational application. But, on the other hand, if we mean sensations of motion when we say ' motor' and refer space perception to these as its coincidental factor, there will be less difficulty in accepting the doctrine. It is quite apparent in the experiments which I have described that the sensations of motion are the variants with the differences of effects and we may well attribute this difference of effect to the action of those conditions which discover the existence of motional effects in consciousness as the varying factors in the phenomena to be explained. But if we are to import into the issue the implications of contrast with the sensory which the term ' motor' so often has, we only introduce confusion into the problem. We have in fact a case where, when that contrast is excluded, there is identity with the sensory theory at large instead of opposition, so that all the controversies connected with the matter are largely the proverbial dispute about words, except that the illusion is not wholly verbal.

James H. HysLOP.

WOODSTOCK, VHRMONT.

\section{DR. BOSANQUET ON IMITATION AND SELECTIVE THINKING.}

In my note in the November number of the Rkvirw, discussing Dr. Bosanquet's criticism, I expressed the intention of continuing the topic - especially with reference to his criticism of the theory of selective thinking. The criticism in question occurs in his discussionwhich was taken up for reply in my earlier note - in the PsychoLogicax REvirw for July. Having in mind the general considerations advanced, as to the need of a thoroughgoing application of the genetic method, I make the following points, dealing rather with the adequacy of the theory of selective thinking, the necessity of some such genetic account being assumed.

On p. $3^{86}$ of his article, Dr. Bosanquet makes the criticism of

'A continuation of the discussion in the November REvrew. 